\title{
Temperature dependence of density of states near the Fermi level in a strain-free epitaxial film of the hole-doped manganite $\mathrm{La}_{0.7} \mathrm{Ca}_{0.3} \mathrm{MnO}_{3}$
}

\author{
J. Mitra, Mandar Paranjape, and A. K. Raychaudhuri \\ Department of Physics, Indian Institute of Science, Bangalore 560 012, India \\ N. D. Mathur and M. G. Blamire \\ Department of Materials Science and Metallurgy, Cambridge University, Cambridge CB2 3QZ, United Kingdom
}

\begin{abstract}
Scanning tunneling spectroscopy measurements were performed on an epitaxial thin film of $\mathrm{La}_{0.7} \mathrm{Ca}_{0.3} \mathrm{MnO}_{3}$ grown on a $\mathrm{NdGaO}_{3}$ substrate. The temperature variation of the density of states (DOS) close to the ferromagnetic transition temperature $\left(T_{c}\right)$ was investigated. The strain-free film exhibiting a sharp metal-insulator transition at $T_{p} \approx T_{c} \approx 268 \mathrm{~K}$ shows no phase separation as seen by the conductivity map, allowing unambiguous determination of the tunneling spectra as a function of $T$. The temperature dependence of the conductance $(d I / d V)$ curves and the normalized DOS clearly indicate a depletion in DOS near $T_{c}$, which fills up as the sample is cooled below $T_{c}$. The metal-insulator transition at $T_{c}$ also shows up in the bias dependence of the tunneling curve as the temperature is changed across the transition. In the metallic phase we find that the DOS is similar to what is expected in a correlated metal.
\end{abstract}

\section{INTRODUCTION}

The field of hole-doped manganites is a topical area of investigation that has been enriched in the past decade with novel experimental results and interesting theoretical insights. ${ }^{1}$ However, understanding the various facets (including the colossal magnetoresistance phenomenon) of the highly complex system is far from complete. Above a critical doping these manganites show a ferromagnetic (FM) to paramagnetic (PM) phase transition at the transition temperature $T_{c}$. Above $T_{c}$ the PM phase exhibits an activated transport, which is believed to be governed by polarons ${ }^{2}$ due to strong electron-phonon coupling, mediated by Jahn-Teller distortion around the trivalent $\mathrm{Mn}^{3+}$ ion. Below $T_{c}$ the ground state is FM metallic $(d \rho / d T>0)$. The transition from the activated PM state to the FM metallic state at $T_{c}$ is often referred to as the metal-insulator transition (MIT). While in many oxides, the temperature-driven MIT is well known, this particular MIT is unusual because, unlike most other oxides, here the high-temperature phase shows activated transport while the low-temperature phase is metallic. Different experiments like optical conductivity ${ }^{3,4}$ and tunneling experiments ${ }^{5-7}$ show that the nature of this "metallic" state below $T_{c}$ is not like that of a conventional metal. However, specific heat measurements ${ }^{8,9}$ at low temperatures show a linear temperature-dependent term as in a conventional metal. A newer feature observed in these oxides is the coexistence of carrier-rich FM metallic and carrier-deficient non-FM insulating regions (often assuming mesoscopic dimensions) that is generally termed electronic phase separation (PS). Both theoretical calculations and experimental reports have shown the existence of PS in this class of materials. ${ }^{10}$ The presence of such insulating states in a FM metallic background has been imaged using local probe measurements like scanning tunneling microscopy ${ }^{11,12}$ (STM) and magnetic force microscopy. ${ }^{13} \mathrm{PS}$ is observed not only near $T_{c}$ but far deeper into the FM metallic state. ${ }^{12}$
In this investigation we address specifically the issue of the sample density of states (DOS) near the Fermi level $\left[N_{s}\left(E_{F}\right)\right]$ as seen by scanning tunneling spectroscopy (STS). The fact that the FM transition is associated with a MIT raises the question whether $N_{s}\left(E_{F}\right)$ changes across the $T_{c}$. Now, the conductivity of the sample is given by $\sigma \simeq e^{2} N_{s}\left(E_{F}\right) D$ (where $D$ is the electron diffusivity). Thus, a low conductivity implies a depleted DOS at $E_{F}$, compared to that in a high-conductivity region. Earlier STS measurements ${ }^{14}$ done on similar systems have shown that $N_{s}\left(E_{F}\right)$ builds rapidly as the temperature is lowered below $T_{c}$. This indicates that the large increase in the $\sigma$ of the system on cooling below $T_{c}$ can be attributed partly to the rapid increase of $N_{s}\left(E_{F}\right)$. The main motivation of the present study is to investigate the temperature dependence of STS in a sample that does not show much evidence of PS. The presence of PS leads to ambiguities in the determination of $N_{s}\left(E_{F}\right)$ since the two coexisting phases (having different conductivities) show different tunneling spectra, resulting in spatially dependent STS. Thus it is desirable to investigate STS in a system that has no PS.

In this paper we report STS measurements carried out as a function of temperature, across $T_{c}$, on a strain-free epitaxial thin film of $\mathrm{La}_{0.7} \mathrm{Ca}_{0.3} \mathrm{MnO}_{3}$ (LCMO) grown on a latticematched substrate $\mathrm{NdGaO}_{3}$ (NGO). The LCMO film, which has large atomically smooth terraces $(\sim 360 \mathrm{~nm}$ wide and several micrometers long), allows STS investigation without the complication of PS and influence of structural discontinuities like grain or platelet boundaries.

\section{EXPERIMENTAL DETAILS}

The $50 \mathrm{~nm}$, (001)-oriented LCMO film was grown on the NGO substrate, using pulsed laser deposition. The details of the growth are given elsewhere. ${ }^{15}$ The film was characterized by $\mathrm{x}$-ray diffraction (XRD) to estimate the strain in the 

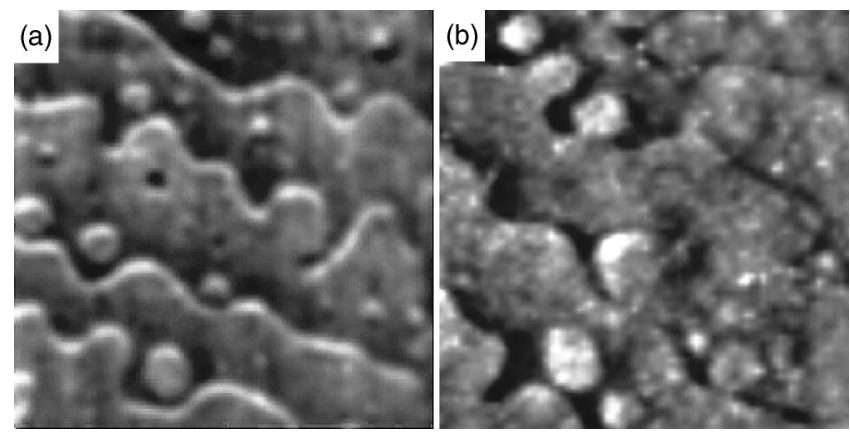

FIG. 1. Topography images of LCMO/NGO. (a) $300 \mathrm{~K}$ $[(\Delta x \times \Delta y \times \Delta z)](1.2 \mu \mathrm{m} \times 1.2 \mu \mathrm{m} \times 0.5 \mathrm{~nm})$ and (b) $100 \mathrm{~K}$ $(1 \mu \mathrm{m} \times 1 \mu \mathrm{m} \times 0.6 \mathrm{~nm})$.

film. Resistivity $(\rho)$ measurements, in the standard fourprobe dc technique, were carried out at 0 and $6 \mathrm{~T}$ magnetic field. Earlier, transport as well as STM and scanning tunneling potentiometry related studies have been carried out on the same sample. ${ }^{16}$ The STM and STS measurements were carried out in a home-made variable-temperature STM using a Pt-Rh tip. The STM is capable of operating from 4.2 to 350 $\mathrm{K}$ in a high-vacuum environment. In operation the sample temperature was kept above room temperature while the cryostat was slowly cooled to prevent contamination of the sample surface. With a fixed tip-sample distance, the STS $I-V$ curves were taken over 5-10 cycles, which were averaged and numerically differentiated to obtain the $d I / d V-V$ curves.

The conductance maps were taken simultaneously with topography images at various temperatures. While taking the conductance maps the dc bias was modulated with a small ac signal and applied to the sample. A lock-in detection of the current amplifier output at the ac modulation frequency gives us a direct measure of the conductance $(d I / d V)$ at fixed dc bias over the whole scan range. The typical ac modulation was kept at 2-4\% of the dc signal. An optimum $100 \mathrm{mV} \mathrm{dc}$ bias was used since in this range the DOS changes substantially at the MIT. This, therefore, enhances the contrast of the conductance maps. At the same time the bias is high enough to sustain a measurable tunnel current at the MIT temperature. At higher biases the relative change in the $d I / d V$ and thus the DOS decreases as the temperature is varied.

\section{RESULTS}

Figures 1(a) and 1(b) show the topography images taken at 300 and $100 \mathrm{~K}$ with $100 \mathrm{mV}$ bias and $0.5 \mathrm{nA}$ tunnel current. The images show that the film has a very clear step and terrace structure which spans the complete range of the image. The steps have a height of $0.4 \mathrm{~nm}$, which is the same as the out-of-plane lattice constant of $0.39 \mathrm{~nm}$, as determined by XRD. The terraces are $\sim 360 \mathrm{~nm}$ wide with a rms roughness of $\sim 0.03 \mathrm{~nm}$. The presence of such smooth terraces allows local tunneling experiments in regions that are unaffected by the presence of steps or grain boundaries and their associated local disorders and nonuniformities. The terrace and step growth seen in this film is a clear signature of nearly uniform strain that is sustained up to a critical thickness depending on the lattice mismatch with the substrate. Typically for films with thickness more than $50 \mathrm{~nm}$ one observes strain relaxation that leads to breakdown of the terraces to platelets and makes the surface rough. ${ }^{16,17}$ From the XRD data we could place a limit on the out-of-plane strain $\epsilon_{z z} \leqslant 0.02 \% .^{16}$ The film, due to its excellent lattice match with the substrate, has negligible strain, which we believe is responsible for showing a rather uniform conductance map. This film is in sharp contrast to the films used in the previous STS investigations where the films were grown on $\mathrm{SrTiO}_{3}, \mathrm{LaAlO}_{3}$ (LAO), or MgO substrates. ${ }^{6,11,12}$ These films had granular or platelet structure (maximum grain size $\sim 50 \mathrm{~nm}$ ) and roughness greater than $1 \mathrm{~nm}$. In a previous STS investigation on $\mathrm{La}_{0.8} \mathrm{Ca}_{0.2} \mathrm{MnO}_{3}$ film by our group ${ }^{14}$ the film used was grown on LAO and also had a terrace pattern although the terrace sizes were much smaller and within a few tens of nanometers. We emphasize that the film quality and presence of strain make a difference in the STS studies. The effects of strain and microstructure in LCMO films grown on different substrates have been investigated before. ${ }^{16}$

Figure 2 shows the resistivity data for LCMO in 0 and $6 \mathrm{~T}$ magnetic field along with the calculated magnetoresistance. It has a PM insulator-FM metallic phase transition with $T_{p} \approx T_{c} \sim 268 \mathrm{~K}$ and remains metallic with a residual resistivity $\rho_{4.2 \mathrm{~K}}=216 \mu \Omega \mathrm{cm}$. $\left(T_{p}\right.$ is the temperature where $d \rho / d T=0$.) The sharpness of transition can be seen from the derivative of the resistivity $(d \rho / d T) /(\rho / T)$, shown in the inset of Fig. 2. The full width of the transition is around $\Delta T \approx 17 \mathrm{~K}$. The low value of residual resistivity and the sharpness of the MIT near $T_{c}$ also reflect the quality of the film.

\section{A. Conductance maps}

In order to establish that such a strain-free film does not show much PS we recorded spatially resolved conductance maps (simultaneously with topography) as a function of temperature (200-300 K). Figure 3(a) shows the topography image and Fig. 3(b) shows the corresponding conductance map at $265 \mathrm{~K}$ (a temperature just below $T_{c}$ where the PS is expected to be maximum ${ }^{11}$ ). In the conductance map image white corresponds to the highest conductance while black indicates the value of lowest conductance. The scale of the conductance range in Fig. 3(b) is $4 \mathrm{nS}$. Features in the conductance maps were seen to correspond to the underlying topographic features to a certain extent mainly near the terrace edges. To investigate the presence of microscopic phase separation in the sample we took line scans across the conductance maps (restricting the lines of scan along single terraces, avoiding the sudden changes in conductance observed at the terrace edges or steps). Figure 3(c) shows the line scan along the line marked on the conductance map. The line scan clearly shows the absence of any phase-separated conducting and insulating states in the system. The fluctuations are purely random and the power spectra do not confirm the presence of any preferred length scales in the conductance map. Thus the absence of PS in the film makes it ideal for STS study since it implies uniform tunneling curves across the film. 


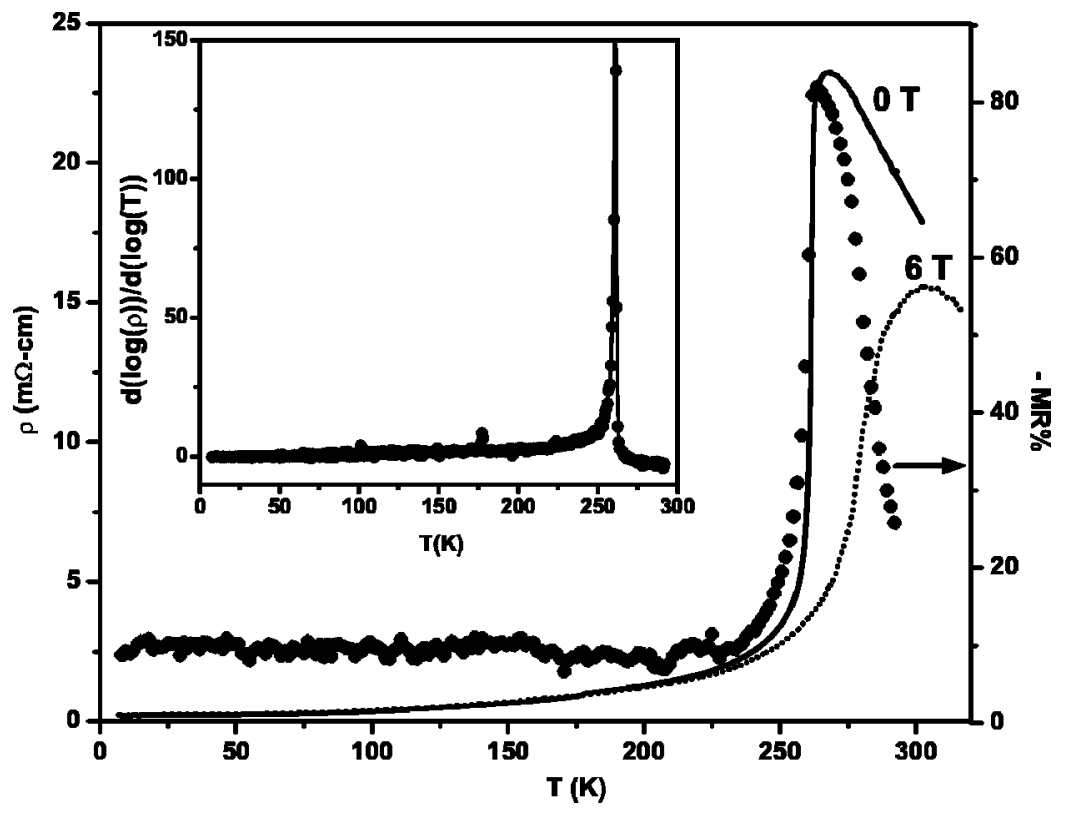

FIG. 2. $\rho$ vs T for LCMO/NGO for 0 and $6 \mathrm{~T}$ magnetic field and MR\%. Inset: sharpness of MIT from $0 \mathrm{~T}$ resistivity data.

B. Scanning tunneling spectroscopy

Figure 4 shows few representative $I-V$ curves taken on LCMO as a function of temperature. The variation of the $I-V$ curve as the temperature changes is apparent from the figure. Close to $T_{c}$ we can see a strong suppression of the tunneling current at low bias $(V<0.3 \mathrm{~V})$ which quickly builds up as the temperature moves away from $T_{c}$ (see Fig. 4 inset). The strong suppression of the tunneling current close to $T_{c}$ (as seen below) is indicative of a strong suppression of the DOS close to the $E_{F}$. This basic feature is in conformity with earlier STS studies. ${ }^{14}$ Figure 5 shows selected $d I / d V-V$ data from 170 to $320 \mathrm{~K}$. The $d I / d V$ data are plotted on a logarithmic scale in order to accentuate the low-bias region, which if plotted in linear scale may lead to an impression of a gap opening up in the tunneling curve close to the transition temperature. This is illustrated in Fig. 6. From $170 \mathrm{~K}$ as the temperature nears $T_{c}$, around $230 \mathrm{~K}\left(T / T_{c}\right.$ $=0.85)$, the zero-bias conductance $\left(G_{0}\right)$ starts decreasing rapidly and reaches a minimum near $260 \mathrm{~K}$, though remaining finite (see Fig. 7). At each temperature the $I-V$ curves were taken at 4-5 places over the sample and were found to give similar conductance spectra obtained with the conductance map within experimental errors.

The estimation of the DOS $N_{s}(E)$ from tunneling spectra is not a trivial issue, particularly when the DOS has a strong dependence on energy as well as temperature, which we dis-
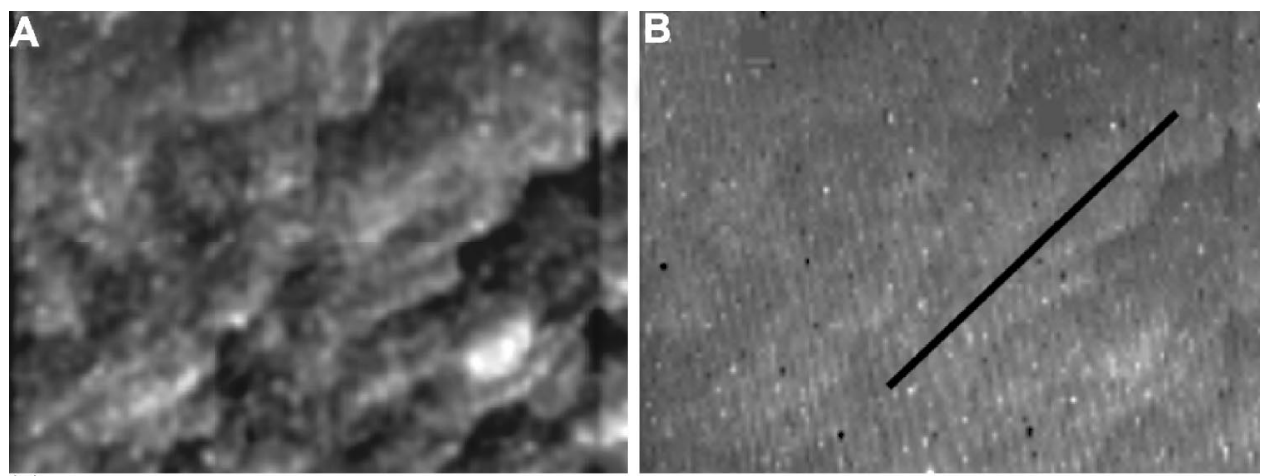

(a)

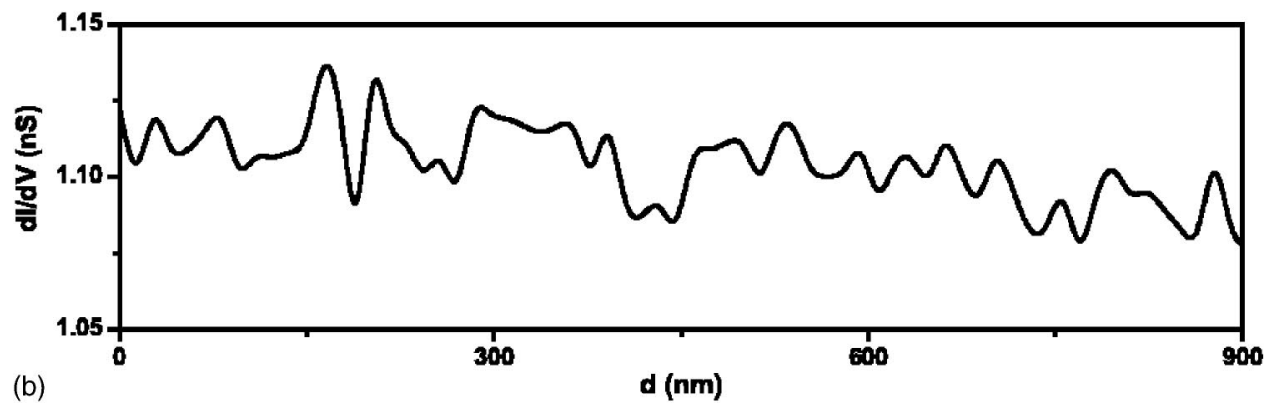

FIG. 3. Simultaneous (a) topography $\quad(1.5 \mu \mathrm{m} \times 1.0 \mu \mathrm{m}$ $\times 0.7 \mathrm{~nm})$ and (b) conductance map $(1.5 \mu \mathrm{m} \times 1.0 \mu \mathrm{m} \times 4 \mathrm{nS})$ with $100 \mathrm{mV}$ dc bias at $265 \mathrm{~K}$. (c) The line scan along a terrace of the conductance map. 


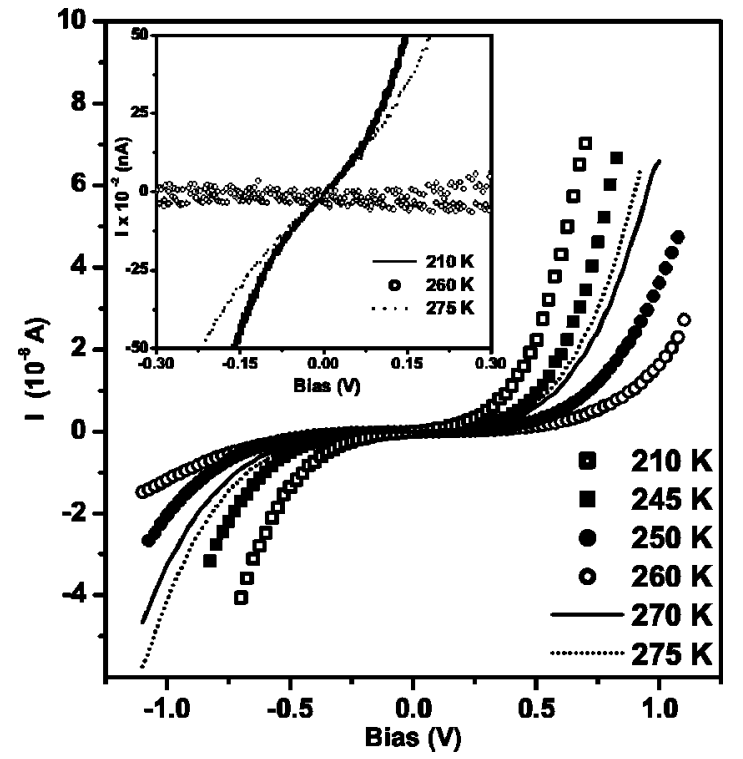

FIG. 4. Bare STS $I-V$ at selected temperatures. Inset shows a closeup of $I-V$ curves at 200,265 , and $275 \mathrm{~K}$ for $V< \pm 0.3 \mathrm{~V}$.

cuss in the next section. However, even without an extensive analysis one can reach certain inferences directly from observation of the tunneling data themselves (Fig. 5). In contrast to a conventional metal (e.g., Au, Pt, etc.) the tunneling curves are strongly biased as well as temperature dependent. These dependencies are more severe in the low-bias region $(V<0.3 \mathrm{~V})$ implying that it is the region close to $E_{F}$ that undergoes maximum change as the temperature is varied. We also find that the change is maximum close to $T_{c}$. At higher biases $(|V|>0.5 \mathrm{~V})$ the temperature dependence is less severe. The strong depression of the tunneling curve close to $T_{c}$ at low bias clearly indicates a strong depression of the DOS closer to $E_{F}$. This can be interpreted as the appearance of a pseudogap. At lower temperatures as the metallic state is stabilized the strong depression in DOS at low bias recovers. We discuss this later in the text. (Note that at much lower temperature $T<40 \mathrm{~K}$ at a bias less than $15 \mathrm{meV}$ a pseudogap again opens up. ${ }^{7}$ ) We also find that the tunneling curves are substantially asymmetric. This is not unexpected because the positive bias represents a contribution from the unfilled states while the negative bias represents that from the filled states. There are no a priori reasons to expect that they are same.

\section{DISCUSSION}

To extract $N_{s}(E)$ from the STS data and observe its variation with temperature we need a normalization technique that estimates the barrier contribution. There are different schemes ${ }^{18,20,21}$ to do that although none can be claimed to be foolproof, particularly when the DOS changes rapidly with temperature and no prior estimate of $N_{s}(E)$ exists. We analyze our experimental data using these schemes and would like to see whether we can reach certain inferences regarding the variation of the DOS at the MIT close to $T_{c}$.

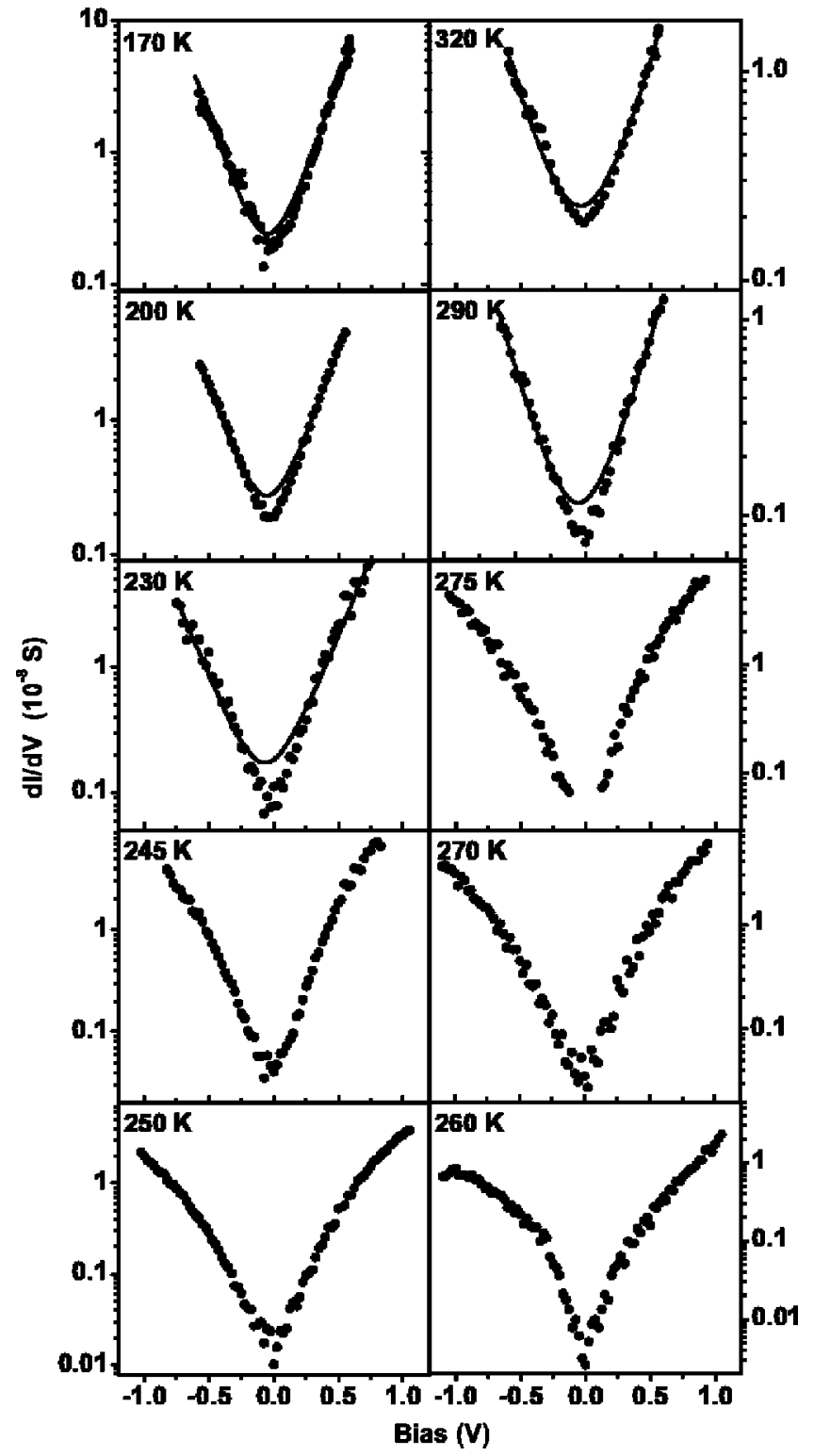

FIG. 5. Variation of $d I / d V-V$ with $T$ from 170 to $320 \mathrm{~K}$. The solid lines are the calculated barrier contribution, fits to Eq. (4).

\section{A. Low-bias region: Signatures of a correlated metal}

In this section we analyze the low-bias region $(V<0.2 \mathrm{~V})$ which shows the maximum change close to $T_{c}$. We recall the expression of the tip-sample tunnel current, across a barrier. In the symmetric form it is given by ${ }^{18}$

$$
\begin{aligned}
I(s, V, W, T)= & c \int_{-\infty}^{\infty} N_{s}\left(E+\frac{e V}{2}\right) N_{t}\left(E-\frac{e V}{2}\right) \bar{t}(s, E, W) \\
& \times\left[f\left(E-\frac{e V}{2}\right)-f\left(E+\frac{e V}{2}\right)\right] d E,
\end{aligned}
$$

where $E$ is the energy of the tunneling electron, $c$ is a constant dependent on the tip-sample effective junction area, $s$ is the tip-sample distance, $W$ is the average work function of the tip and sample surface, $V$ is the applied bias between the tip and the sample, $T$ is the temperature, $N_{t}(E)$ and $N_{s}(E)$ are 


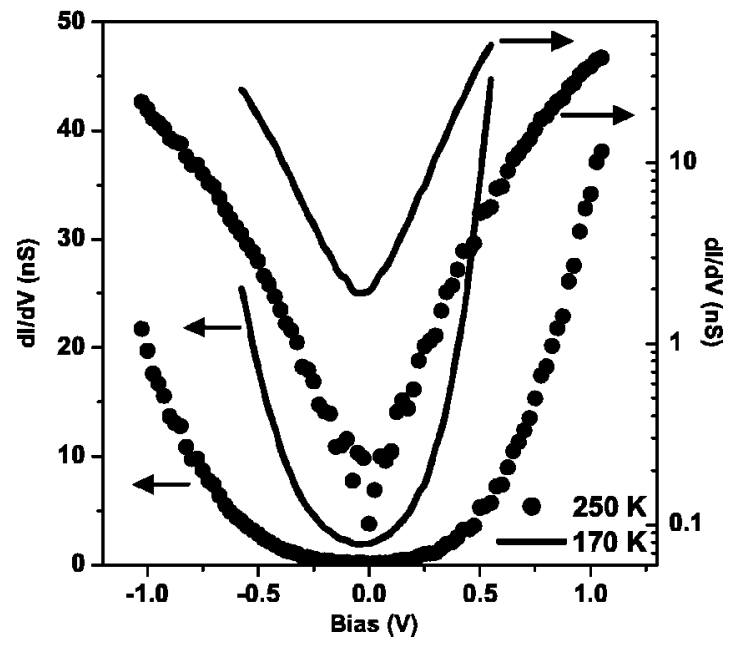

FIG. 6. $d I / d V-V$ at 170 and $250 \mathrm{~K}$. Plotted in linear (left) and logarithmic (right) scale. The graph shows that in the linear scale the $d I / d V$ gives the impression of a gap.

the tip and sample DOSs, $f(E)$ is the Fermi function at temperature $T$, and $\bar{t}$ is the barrier penetration factor. We assume a one-dimensional trapezoidal barrier and estimate $\bar{t}$ via a WKB semiclassical calculation. This gives $\bar{t}(s, E, W)=\exp [-2 k s \sqrt{2(W-E)}]$, where $k=\sqrt{m} / \hbar$. The typical $W$ is well above $1 \mathrm{eV}$. Thus, for low biases the barrier function $\bar{t}(s, E, W)$ is essentially governed by $W$ and is nearly independent of the bias $V=E / e$ In that case, $\bar{t}(s, E, W)$ can be taken outside the integral. Assuming a flat $N_{t}(E)$, in the energy scale used by us we find that $d I / d V \propto N_{s}$. Thus in the low-bias region the bias dependence of $d I / d V$ will reflect the energy dependence of $N_{s}\left(E-E_{F}\right)$. We find that in this region the bias dependence of $d I / d V(V)=G(V)$ follows a power law,

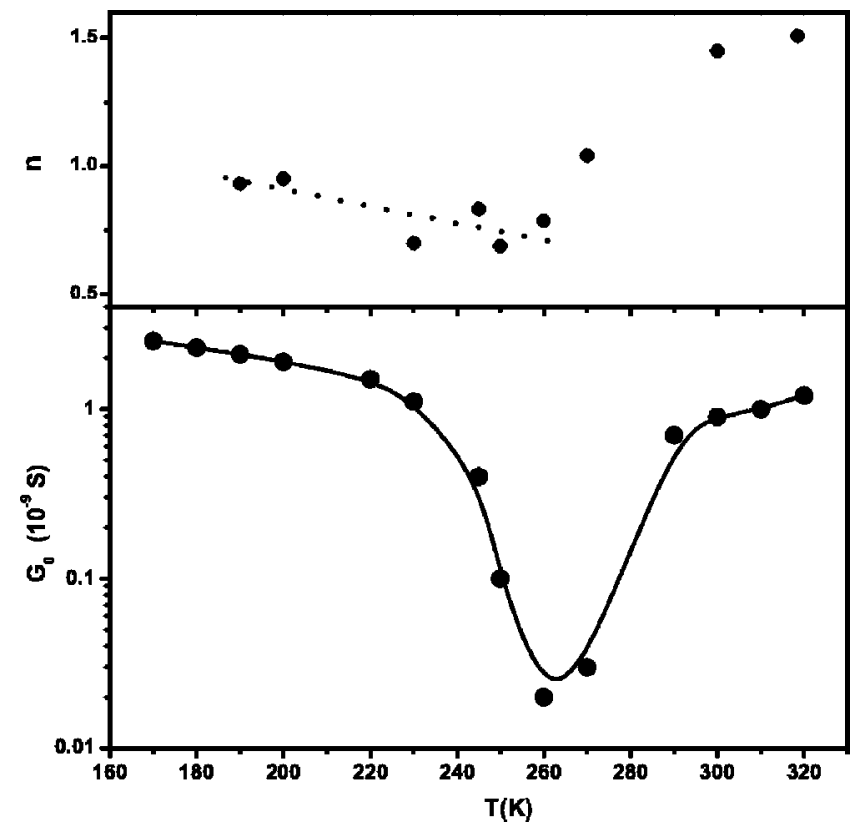

FIG. 7. Variation of $n$ and $G_{0}$ with $T$. $G_{0}$ shows a variation of two orders of magnitude near $T_{c}$ (see text for details).

$$
G(V)=G_{0}\left[1+\left(\frac{|V|}{V^{*}}\right)^{n}\right]
$$

Here $V^{*}$ is a constant. $n$ and $V^{*}$ are determined from the fit of the conductance data to Eq. (2). The exponent $n$ as well as $G_{0}$ have interesting temperature dependence. [Note that the tunneling curves are asymmetric at all temperatures. We took the symmetric part defined as $G_{s}=[G(+V)+G(-V)] / 2$ and obtained the parameters in Eq. (2).] Figure 7 shows the variation of $n$ and $G_{0}$ with temperature. Below $T_{c}$, on the metallic side, $n$ has a shallow variation with temperature and always remains less than 1 . It slowly decreases as the $T_{c}$ is approached from below. $G_{0}$ shows a shallow variation for $170<T<230 \mathrm{~K}$ after which it undergoes a large drop, reaching a minimum near $T_{c}$. This is similar to what has been observed in a barrier-type tunnel junction. ${ }^{7}$ The observed tunneling conductance $G(V)$ being roughly proportional to $N\left(E-E_{F}\right)$, the dependence of $G(V)$ on $V$ as expressed through Eq. (2) can be interpreted as $N\left(E-E_{F}\right)$ following the relation

$$
N\left(E-E_{F}\right)=N\left(E_{F}\right)\left[1+\left(\frac{\left|E-E_{F}\right|}{\Delta}\right)^{n}\right],
$$

where $\Delta=e V^{*}$ is a constant, associated with a pseudogap, and $N\left(E_{F}\right)$ is the DOS at the Fermi level. This dependence of the single-particle DOS on $n \approx 0.5$ is expected in a disordered metal with electron-electron interaction. ${ }^{22}$ Such an energydependent DOS near $E_{F}$ has been seen in a number of correlated oxides. ${ }^{19,23,24}$ Interestingly the $n$ in all of them is less than 1 and it evolves from 0.5 to 1 as the MIT is approached. The typical $\Delta \sim 100 \mathrm{meV}$ near $T_{c}$. Above $T_{c}, n$ rises rapidly to 1.5 accompanied by a rapid buildup in $G_{0}$. This signifies the termination of the sharply temperaturedependent DOS close to $T_{c}$. The important point to note is that the temperature dependence of $G(V)$ reflects the MIT at $T_{c}$ rather distinctly, through the variation of both $G_{0}$ as well as $n$.

\section{B. Normalizing the DOS using barrier parameter $\bar{t}(s, E, W)$}

Equation (1) can be used to deconvolute the effect of the barrier parameter $\bar{t}(s, E, W)$. The procedure for that has been explained extensively before. ${ }^{18,14,7}$ One can deduce the differential tunneling conductance from Eq. (1) as

$$
\frac{d I_{\text {const }}^{0}}{d V}=\sigma_{\text {const }}^{0}(s, V)=\frac{c}{2} N_{t} N_{s}[\bar{t}(s, e V / 2, W)+\bar{t}(s,-e V / 2, W)]
$$

assuming a constant $N_{t}, N_{s}$ and $k_{B} T \ll e V$. A fit of the bare conductance data with Eq. (4) essentially estimates the barrier function $\bar{t}$. Thus normalizing the bare $d I / d V$ by the estimated $\bar{t}$ removes the barrier effect and gives us a normalized conductance. Since $N_{t}$ is flat ${ }^{31}$ in this energy window $( \pm 1$ $\mathrm{eV})$ the observed energy dependence of the normalized conductance arises solely from the sample. The fit further provides us with values for the average work function and the tip-sample distance that characterizes the junction. Typically 


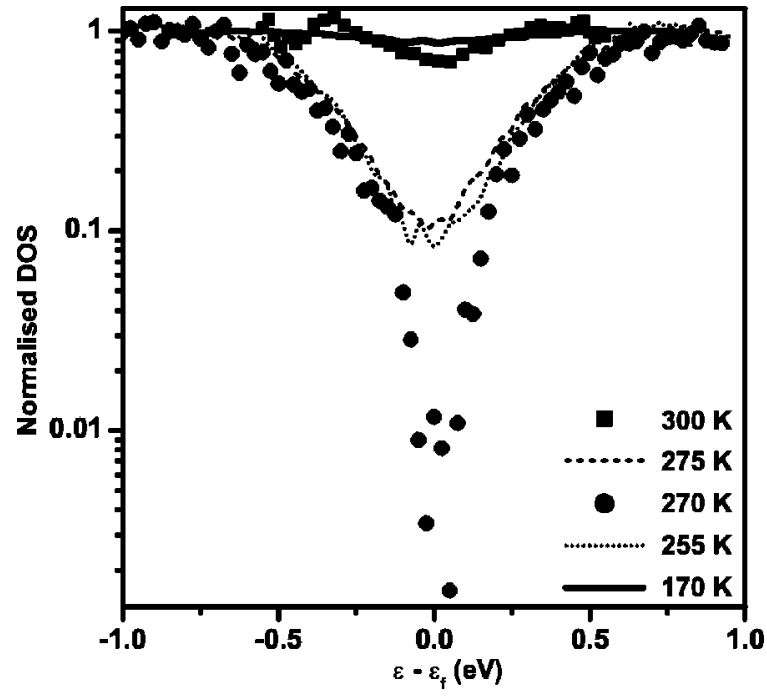

FIG. 8. Evolution of the normalized DOS with $T$. DOS is seen to be heavily depleted near $T_{c}(270 \mathrm{~K})$. It is almost flat at $170 \mathrm{~K}$ and above $T_{c}(300 \mathrm{~K})$ a depletion near $E_{F}$ is retained.

$W \approx 1.5 \mathrm{eV}$ and the tip-sample distance estimated from the fit is $\approx 7 \AA$.

The solid lines shown in Fig. 5 are the fit of the conductance data to the above Eq. (4). They show the bias dependence that arises only from the barrier factor if $N_{s}$ is flat. As seen in the figure, for $T \ll T_{c}$ the above equation fits the conductance curves. This reflects on the metallic nature of LCMO at low temperatures. As the temperature approaches $T_{c}$ we see a deviation of the $d I / d V$ curves from the conventional metallic tunneling behavior and are seen to be dominated by strongly energy-dependent features of $N_{s}$, particularly near the zero bias. This is evident for the conductance curves in the temperature interval $230<T<270 \mathrm{~K}$. In this temperature interval we have restricted the fitting [to Eq. (4)] only to the high-bias region $(> \pm 0.5)$ to get an estimate of the contribution from the barrier.

The deconvolution method of obtaining the DOS gives the correct energy dependence of $N_{s}(E)$ but not the correct absolute value of the same. The limitations of this technique are discussed elsewhere. ${ }^{18}$ Figure 8 shows the normalized $N_{s}(E)$ calculated from the $d I / d V$ data and its variation with temperature. As we approach $T_{c}$ a large depletion in the DOS is seen with a pseudo-gap-like feature opening in the calculated DOS at $270 \mathrm{~K}$. This observation is very similar to what has been stated in the previous section. We can conclude that even after deconvoluting the barrier factor the strong bias dependence near the low-bias region seen in the bare tunneling data (Fig. 5) stays and is nearly independent of the deconvolution details. For $T \ll T_{c}$, well within the metallic state, $n$ being higher, the DOS shows much less depression. Above $T_{c}$ the depletion is "filled" to a large extent but a small depression still remains at $E_{F}$ at the high temperatures, as is evident from the normalized DOS at $320 \mathrm{~K}$.

Interestingly these STS conductance data and the calculated DOSs showed that a depletion in the DOS starts much below $T_{c}$ or $T_{p}$. Although the macroscopic resistivity data

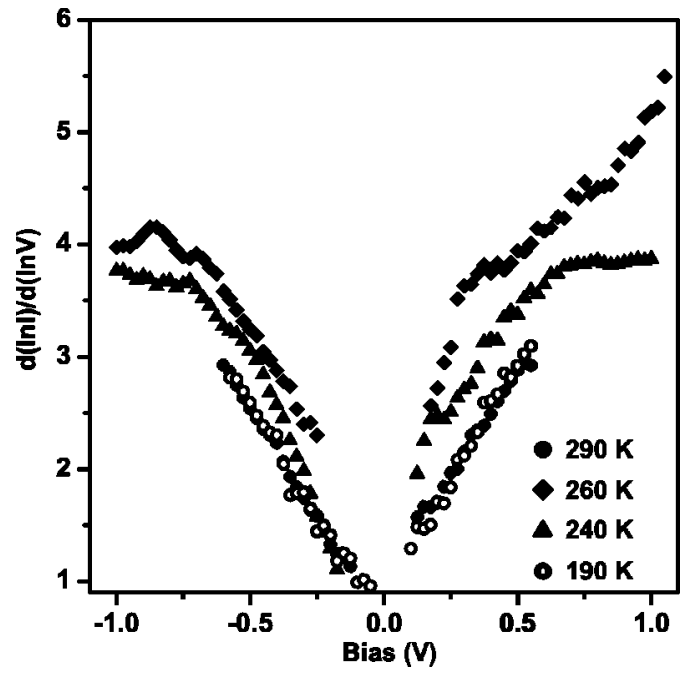

FIG. 9. $d(\ln I) / d(\ln V)-V$ curves for LCMO at different temperatures.

indicate that the MIT temperature is almost concurrent with $T_{c}$ and is rather sharp, the DOSs obtained from the STS data suggest that a deviation from metallic tunneling behavior start $\sim 25 \mathrm{~K}$ below $T_{c}$.

\section{Normalization with $d \ln I / d \ln V$}

A standard practice in analyzing STS data is to normalize the data using logarithmic derivative $d \ln I / d \ln V$. Normalized $(d I / d V)$ by $(I / V)$ can be shown to be proportional to $N_{s}$ under certain approximations. ${ }^{21}$ Thus the voltage dependence of $(d I / d V) /(I / V)$ is expected to represent the energy dependence of the DOS. However, if a gap opens up at $V=0$ one may obtain a divergent logarithmic derivative. For this reason we avoided taking the logarithmic derivative in the bias range where the gap opens up. ${ }^{25}$ In Fig. 9 we show the derivative as a function of the bias at some representative temperatures. $d \ln I / d \ln V$ reaches the limiting value of 1 close to the zero bias. The limiting logarithmic derivative of unity is expected mathematically when there is no real gap at $V=0$. The interesting aspect is the existence of well-defined structures in the normalized DOS in the energy range $\approx 0.5 \mathrm{eV}$. The normalized DOS is similar to that seen previously. ${ }^{14}$ We also find a substantial enhancement of the DOS at higher energy close to $T_{c}$. This would imply that as the spectral weight near the $E_{F}$ decreases near $T_{c}$ there is a shift in the spectral weight to higher bias. The shift of the spectral weight from the $E_{F}$ to higher energy appears to be a recurring feature in the photoelectron spectroscopy of these materials. $^{26}$

\section{Certain other aspects of the DOS as seen in this experiment}

The focus of the discussion in the previous subsections was to explore the change in observable features in the DOS close to $T_{c}$. Data presented above clearly show that there is an evolution in the DOS including a depression close to $E_{F}$ near the MIT. Here we discuss a few other issues that we 
have observed and also put our data in the perspective of other electronic spectroscopic data.

Temperature-dependent studies of the electronic spectroscopy in both $\mathrm{La}_{0.67} \mathrm{Ca}_{0.33} \mathrm{MnO}_{3}$ and $\mathrm{La}_{1-x} \mathrm{Sr}_{x} \mathrm{MnO}_{3}(x \geqslant 0.2)$ have shown that the spectral weight and hence the DOS builds up at the $E_{F}$ below $T_{c}$ although it remains low. ${ }^{27,26}$ The evolution of the DOS seen by us near $T_{c}$ is in close agreement with this observation and once again emphasizes that the spectral evolution near the Fermi level plays an important role in the MIT seen in the manganites. Such a strong dependence of the DOS is a rather unusual phenomenon. We believe that the cause is the fact that the ratio $U_{\text {eff }} / W\left(U_{\text {eff }}\right.$ is the Coulomb interaction strength and $W$ is the bandwidth) is temperature dependent ${ }^{26}$ and decreases on cooling. This is due to the fact that $W$ is dependent on the degree of spin order which itself is a temperature-dependent quantity. $W$ increases on increase of the spin order, which increases in the FM state. $U_{\text {eff }}$ decreases on FM ordering. Decrease of $U_{\text {eff }} / W$ stabilizes the metallic phase although as a correlated metal. When the $T_{c}$ is approached from below, increase in $U_{\text {eff }}$ and decrease in $W$ would remove states from the region close to $E_{F}$ and would move them to higher energy. This is in conformity with what we would like to conclude from the change in DOS observed by us.

The tunneling data further show that there is a low-energy scale below which the tunneling curve becomes strongly temperature dependent $(E<0.5 \mathrm{eV})$. A close look at the photoemission spectra and analysis ${ }^{28-30}$ (in $\mathrm{La}_{1-x} \mathrm{Sr}_{x} \mathrm{MnO}_{3}$ ) shows that the states above $E_{F}$, in this energy range, are the doped hole states which take part in the conduction process. One would expect that to be similar in LCMO also. The strong temperature dependence of the DOS at energies lower than $0.5 \mathrm{eV}$ is likely to arise from the doped hole states at the bottom of the conduction band.

A crucial aspect in our data, also seen in previous reports of STS studies on manganites, ${ }^{14,6}$ is the rise in the zero-bias tunneling conductance $G_{0}$ above the MIT transition temperature. In this region the transport is activated and one would expect to see opening of a gap in the DOS. In contrast, we see a very weak depression in the DOS (see Fig. 8) at $E_{F}$. This observation remains mostly unexplained. However, one may offer the following qualitative explanation for this. These tunneling experiments were carried out at a finite temperature $(200-300 \mathrm{~K})$ that tends to fill up the gap and increase the tunneling conductance near zero bias. Simulation of STS tunneling spectra shows that due to the finitetemperature effect we will observe a finite $G_{0}$, even in the presence of a gap $\sim 100 \mathrm{meV}$. In the metallic phase $\left(T<T_{p}\right)$ also, simulations show that a small gap would give a finite $G_{0}$, due to the finite temperature. Thus on the basis of these tunneling data one cannot completely rule out the presence of a gap.

Alternatively, there may be a finite DOS at and close to $E_{F}$ and what we may have is more like a soft gap. The states in the vicinity of $E_{F}$ are then localized. The activated transport could be like variable-range hopping.

\section{E. Comparison with other experiments}

Previously barrier-type tunneling experiments have been carried out on single crystals of $\mathrm{LCMO},{ }^{7}$ primarily in the low-temperature regime. The conductance data obtained from both the measurements have been analyzed using Eq. (2). The barrier tunneling experiments at $4.2 \mathrm{~K}$ show that the exponent $n \simeq 0.7$ for $100 \geqslant V \geqslant 15 \mathrm{mV}$. This exponent has been observed to persist at $77 \mathrm{~K}$. The conductance spectra, obtained from the STS measurements, below $T_{p}$ are also seen to follow a similar power-law behavior, for $V \sim 100 \mathrm{mV}$, with the exponent $n$ having a shallow variation from 0.9 to 0.6 between $160 \mathrm{~K}$ and $T_{p}$. Together these observations suggest that the low-bias $(V \leqslant 100 \mathrm{mV})$ power-law dependence (with $n<1$ ) is present throughout the FM metallic phase of the system. Such an exponent of less than 1 is quite characteristic of strongly correlated metallic oxides. ${ }^{19}$

We also note that most of the previous STS studies on similar samples have been carried out on samples under large biaxial strain and PS (wherever such investigation was carried out). The initial STS reports of Biswas et al. ${ }^{14}$ and Wei et al. ${ }^{6}$ were on thin films deposited on $\mathrm{LaAlO}_{3}$ which has $\sim 2.3 \%$ lattice-mismatch strain with $\mathrm{La}_{0.7} \mathrm{Ca}_{0.3} \mathrm{MnO}_{3}$. They both showed a DOS with a gap below $T_{p}$, indicative of insulating states. Recently, Fath et al. ${ }^{11}$ and Becker et al. ${ }^{12}$ reported spatially resolved STS images of PS on films of $\mathrm{La}_{0.73} \mathrm{Ca}_{0.27} \mathrm{MnO}_{3}$ and $\mathrm{La}_{0.7} \mathrm{Sr}_{0.3} \mathrm{MnO}_{3}$, respectively. These films deposited on $\mathrm{SrTiO}_{3}$ and $\mathrm{MgO}$ also have large filmlattice mismatch of $\sim-0.6 \%$ and $\sim 8 \%$ and thus were strained. They observed PS for $T \leqslant T_{c}$ with the metallic fraction increasing with application of magnetic field or lowering of temperature. However, even at the highest magnetic field $(9 \mathrm{~T})$ or at the lowest temperature there were small yet finite fractions of insulating phases. Becker et al. ${ }^{12}$ also observed a gap in the $d I / d V$ curves over the insulating regions, which is independent of temperature. However, they did not observe any such gap in the metallic regions. Similar experiments carried out on a thin film of $\mathrm{La}_{0.7} \mathrm{Sr}_{0.3} \mathrm{MnO}_{3}$ deposited on NGO (lattice mismatch $0.23 \%$ ) does not show any PS. ${ }^{32}$ These reports indicate that probably the underlying lattice mismatch strain has a role to play in the PS observed in these films.

\section{CONCLUSION}

To summarize, we present extensive investigation of temperature-dependent STS (in a strain-free film) of $\mathrm{La}_{0.7} \mathrm{Ca}_{0.3} \mathrm{MnO}_{3}$ that shows no phase separation. We did observe a clear signature of the metal insulator transition in the DOS. The energy dependence of the DOS close to $E_{F}$ changes quantitatively across $T_{c}$. Interestingly the energy dependence of the DOS near $E_{F}$, in the metallic state, is similar to that seen in other correlated oxides with disorder. The sharp rise in $\rho$ on approaching $T_{c}$ from the metallic side appears to be determined by a depression in the DOS.

\section{ACKNOWLEDGMENTS}

J.M. acknowledges CSIR (Government of India) for financial support. A.K.R. wants to thank DST (Government of India) for support. The exchange visits were supported by the India-U.K. Technology Fund (D.S.T.). 
${ }^{1}$ CMR, Charge Ordering and Related Properties of Manganese Oxides, edited by C. N. R. Rao and B. Raveau (World Scientific, Singapore, 1998); Colossal Magneto-Resistive Oxides, edited by Y. Tokura (Gordon and Breach Science, Amsterdam, 2000).

${ }^{2}$ M. Jaime et al., Phys. Rev. B 54, 11914 (1996).

${ }^{3}$ Y. Okimoto, T. Katsufuji, T. Ishikawa, A. Urushibara, T. Arima, and Y. Tokura, Phys. Rev. Lett. 75, 109 (1995).

${ }^{4}$ Y. Okimoto, T. Katsufuji, T. Ishikawa, T. Arima, and Y. Tokura, Phys. Rev. B 55, 4206 (1997).

${ }^{5}$ Amlan Biswas and A. K. Raychaudhuri, J. Phys.: Condens. Matter 8, L739 (1996).

${ }^{6}$ J. Y. T. Wei, N. C. Yeh, and R. P. Vasquez, Phys. Rev. Lett. 79, 5150 (1997).

${ }^{7}$ J. Mitra, A. K. Raychaudhuri, Ya. M. Mukovskii, and D. Shulyatev, Phys. Rev. B 68, 134428 (2003).

${ }^{8}$ J. J. Hamilton, E. L. Keatley, H. L. Ju, A. K. Raychaudhuri, V. N. Smolyaninova, and R. L. Greene, Phys. Rev. B 54, 14926 (1996).

${ }^{9}$ T. Okuda, A. Asamitsu, Y. Tomioka, T. Kimura, Y. Taguchi, and Y. Tokura, Phys. Rev. Lett. 81, 3203 (1998).

${ }^{10}$ Elbio Dagotto, Takashi Hotta, and Adriana Moreo, Phys. Rep. 344, 1 (2001).

${ }^{11}$ M. Fath, S. Friesem, A. A. Menovsky, Y. Tomioka, J. Aarts, and J. A. Mydosh, Science 285, 1540 (1999).

${ }^{12}$ T. Becker, C. Streng, Y. Luo, V. Moshnyaga, B. Damaschke, N. Shannon, and K. Samwer, Phys. Rev. Lett. 89, 237203 (2002).

${ }^{13}$ Qingyou Lu, Chun-Che Chen, and Alex de Lozanne, Science 276, 2007 (1997).

${ }^{14}$ Amlan Biswas, Suja Elizabeth, A. K. Raychaudhuri, and H. L. Bhat, Phys. Rev. B 59, 5368 (1998).

${ }^{15}$ M. H. Jo, N. D. Mathur, N. K. Todd, and M. G. Blamire, Phys. Rev. B 61, R14 905 (2000).
${ }^{16}$ Mandar Paranjape, A. K. Raychaudhuri, N. D. Mathur, and M. G. Blamire, Phys. Rev. B 67, 214415 (2003).

${ }^{17}$ R. A. Rao, D. Lavric, T. K. Nath, C. B. Eorn, L. Wu, and F. Tsui, Appl. Phys. Lett. 73, 3294 (1998).

${ }^{18}$ Vladimir A. Ukraintsev, Phys. Rev. B 53, 11176 (1996).

${ }^{19}$ A. K. Raychaudhuri, K. P. Rajeev, H. Srikanth, and N. Gayathri, Phys. Rev. B 51, 7421 (1995).

${ }^{20}$ N. D. Lang, Phys. Rev. B 34, 5947 (1986).

${ }^{21}$ J. A. Stroscio and R. M. Feenstra, in Scanning Tunneling Microscopy, edited by J. A. Stroscio and W. J. Kaiser, Methods of Experimental Physics Vol. 27 (Academic, New York, 1993).

${ }^{22}$ B. L. Altshuler and A. G. Aronov, in Electron-Electron Interactions in Disordered Systems, edited by A. L. Efros and M. Pollak (North-Holland, Amsterdam, 1985).

${ }^{23}$ A. K. Raychaudhuri, Adv. Phys. 44, 21 (1995).

${ }^{24}$ D. D. Sarma et al. Phys. Rev. Lett. 80, 4004 (1996).

${ }^{25}$ This problem is often avoided by taking a weighted average of $(I / V)$ which itself contains adjustable parameters. See Refs. 6 and 21 for details.

${ }^{26}$ T. Saitoh et al., Phys. Rev. B 51, 13942 (1995).

${ }^{27}$ J. H. Park, C. T. Chen, S. W. Cheong, W. Bao, G. Meigs, V. Chakarian, and Y. U. Idzerda, Phys. Rev. Lett. 76, 4215 (1996).

${ }^{28}$ A. Chainani, M. Mathew, and D. D. Sarma, Phys. Rev. B 47, 15397 (1993).

${ }^{29}$ W. A. Harrison, Phys. Rev. 123, 85 (1961).

${ }^{30}$ E. L. Wolf, Principles of Electron Tunneling Spectroscopy (Oxford University Press, New York, 1985).

${ }^{31}$ Assumption of a flat $N_{t}$ for the Pt-Rh tip is justified from the observed DOS for other pure metals, e. g., Au; Philip G. Collins et al., Phys. Rev. Lett. 82, 165 (1999).

${ }^{32}$ R. Akiyama, H. Tanaka, T. Matsumoto, and T. Kawaia, Appl. Phys. Lett. 79, 4378 (2001). 